\title{
Unraveling the Species Dilemma in Capsicum frutescens and C. chinense (Solanaceae): A Multiple Evidence Approach Using Morphology, Molecular Analysis, and Sexual Compatibility
}

\author{
Jit B. Baral and Paul W. Bosland 1 \\ Department of Agronomy and Horticulture, MSC 3Q, New Mexico State University, Las Cruces, \\ NM 88003-8003
}

\begin{abstract}
ADDITIONAL INDEX WORDS. Capsicum chinense, C. frutescens, species, RAPD, polymorphism, multiple evidence, genetic relationships

ABSTRaCt. The species status of two morphologically closely related species, Capsicum frutescens L. and $C$. chinense Jacq., was investigated using typological, phylogenetic, and biological species concepts. Diagnostic morphological differences, randomly amplified polymorphic DNA (RAPD) marker-based cluster analysis, and hybrid analyses were used to delimit $C$. frutescens and $C$. chinense. In many cases two morphological characters, calyx constriction and flower position, can separate accessions of $C$. frutescens from $C$. chinense. The RAPD-based analysis clearly separated accessions of $C$. frutescens and $C$. chinense into two distinct groups. The average genetic similarity within $C$. frutescens and $C$. chinense accessions was 0.85 and 0.8 , respectively, whereas the average genetic similarity between $C$. frutescens and $C$. chinense accessions was only 0.38 . The progenies obtained from $C$. frutescens and $C$. chinense hybridization had reduced fertility. Based on these evidences, $C$. frutescens and $C$. chinense represent two morphologically diagnosable, phylogenetically distinct, and reproductively isolated species.
\end{abstract}

Two Capsicum L. species, C. frutescens and C. chinense, are morphologically similar. One of the most lingering questions in Capsicum taxonomy is whether $C$. frutescens and $C$. chinense are two different species or botanical varieties of the same species. A species is defined as a group of actually or potentially interbreeding natural populations that are reproductively isolated from other such groups (Mayr, 1970). On the other hand, botanical variety is a distinct morphological subgroup within a species. It has been argued that $C$. frutescens and $C$. chinense are separate species (Smith and Heiser, 1957), and conversely that they are both one species, but different varieties (Eshbaugh et al., 1983). However, the genetic relationship between these two species is still in speculation.

Capsicum frutescens has maintained its species recognition since Linnaeus (1753). He described it as having paired pedicels per node and a frutescent stem, i.e., perennial plant habit. Shaw and Khan (1928) added waxy greenish-white corolla as a unique character of $C$. frutescens. In addition, Standley (1931) stated that $C$. frutescens has small fruits and the pedicel is generally longer than the fruit.

The name $C$. sinense (C. chinense) was first given by Jacquin in 1776. But this recognition was unknown until Smith and Heiser (1957) re-examined these materials and suggested separating Jacquin's Capsicum sinense from $C$. frutescens and renaming it as $C$. chinense. Smith and Heiser (1957) described C. chinense as having glabrous leaves and stem, with ovate to ovate lanceolate leaves. There are two to five flower buds per node, pedicels are declinate and relatively short and thick. In addition, it has a calyx without teeth, but with a marked constriction between the pedicel

Received for publication 8 Feb. 2004. Accepted for publication 17 Apr. 2004 A contribution of the New Mexico Agr. Expt. Sta., New Mexico State Univ., Las Cruces.

${ }^{1}$ To whom reprint requests should be addressed. Email: pbosland@nmsu.edu and calyx. However, there are accessions that possess one or a few diagnostic morphological characters from one species and the rest of the characters from the other species, creating difficulties in species assignment. Smith and Heiser (1957) compared these two species systematically for the first time, and concluded that $C$. frutescens and $C$. chinense could be distinguished morphologically. Their hybridization experiment between $C$. frutescens and $C$. chinense resulted in a very low seed set as well as a very low pollen viability in plants of the $F_{1}$ generation. The $F_{1}$ plants produced nonviable seeds. As more accessions of both species were studied, they were found to be closely related. The relationship appeared to be so close that separate species treatment was eventually questioned by many researchers (Eshbaugh, 1993; McLeod et al., 1983; Pickersgill, 1988). The conflicting reports have confounded the biological and taxonomic relationships between these two species.

Capsicum frutescens and $C$. chinense are sympatrically distributed throughout their range in Peru, Brazil, Colombia, and Bolivia (Pickersgill, 1971). Anumerical taxonomic analysis using morphological characters could not separate the accessions of $C$. frutescens and $C$. chinense according to species (Pickersgill et al., 1979). Similarly, use of various enzyme loci also could not distinguish $C$. annuum, $C$. frutescens, and $C$. chinense accessions (Jensen et al., 1979). Egawa and Tanaka (1984) reported fertile interspecific hybrids between $C$. frutescens and $C$. chinense with regular meiosis.

Herein, we describe a more comprehensive examination of $C$. frutescens and $C$. chinense using morphological, phylogenetic, and sexual compatibility as evidence. The morphological, phylogenetic, and sexual compatibility approaches correspond with typological, phylogenetic, and biological species concepts, respectively. Morphological characters were examined to determine whether fixed differences exist between $C$. frutescens and C. chinense (typological species concept). Randomly ampli- 
fied polymorphic DNA (RAPD) marker-based cluster analysis determined if $C$. frutescens and $C$. chinense are two separate species, based on the phylogenetic species concept. The sexual compatibility between $C$. frutescens and $C$. chinense was investigated to understand whether $C$. frutescens and $C$. chinense are reproductively isolated (biological species concept).

\section{Materials and Methods}

Plant materials. A total of 191 accessions of $C$. frutescens and 223 accessions of $C$. chinense were acquired from the USDA, Regional Plant Introduction Station, Griffin, Ga., and 20 accessions of $C$. frutescens were obtained from the New Mexico Capsicum Accession (NMCA) collection at New Mexico State Univ. These accessions represent collections from various regions of the World.

MoRPHOLOGY. Morphological data were collected from plants in field plots for the following diagnostic morphological characters: leaf texture, number of flowers per axil, flower position, calyx margin, calyx constriction, corolla color, anther color, filament color, fruit persistence, and fruit size. The morphological data from the field observations were analyzed by discriminant function analysis (SAS Institute, 2001) to determine if any combination of characteristics could separate these two species.

RAPD ANALYSIS. RAPD produced polymorphic DNA markers. Accessions were selected for RAPD analysis based on morphological characterizations. Plants fitting the current taxonomic description of $C$. frutescens and $C$. chinense and showing uniformity in the field were selected for RAPD analysis. Among the 75 accessions selected for the analysis, 41 accessions were $C$. frutescens and the other 34 accessions were $C$. chinense. In addition, 'Greenleaf Tabasco', an interspecific hybrid between $C$. frutescens and $C$. chinense, was also included. This accession possesses most of the characters of $C$. frutescens, but contains some features, such as a constricted calyx and virus resistance from $C$. chinense (Greenleaf et al., 1970). One accession of $C$. annuum L. was used as an outgroup. A list of accessions including the PI number and the country of origin is provided in Table 1.

DNA isolation. From each accession, six plants were randomly selected. Collection of tissue samples, DNA isolation, and DNA quantification methods were as described by Votava and Bosland (2002). After quantification, the DNA was diluted to $10 \mathrm{ng} \cdot \mathrm{mL}^{-1}$ in a TE buffer (10 $\mathrm{mm}$ Tris and $0.1 \mathrm{~mm}$ EDTA) for further use.

PCR FOR RAPD ANALYSIS. The cellular DNA was amplified using the polymerase chain reaction method (PCR) to generate RAPD markers (Williams et al., 1993). PCR was performed on a Geneamp 9600 PCR system (Perkin Elmer Corp., Foster City, Calif.). The decamer primers obtained from Operon Technology (Alameda, Calif.) were diluted in distilled water. Primers used in this analysis were: OPAA-11, OPE-05, OPM-04, OPA-04, OPA-07, OPE-12, OPC-06, OPA-16, OPE-02, OPA-17, OPF-05, OPM-09, and OPA-02. The total volume of each PCR reaction was $20 \mathrm{~mL}$ containing: $20 \mathrm{ng}$ template DNA, $10 \mathrm{~mm}$ Tris- $\mathrm{HCl}, 10$ mм KCl, 356 mм $\mathrm{MgCl}_{2}$, 101 mm each dNTP, 2 units AmpliTaq polymerase (Stoeffel fragment), and $0.24 \mathrm{~mm}$ primer.

The PCR thermal profile was as described by Gallego and Martínez (1997). There was a preliminary step of 2 min at $94{ }^{\circ} \mathrm{C}$ for the template denaturation. The first 10 cycles consisted of $30 \mathrm{~s}$ at $94^{\circ} \mathrm{C}, 1 \mathrm{~min}$ at $55^{\circ} \mathrm{C}$, and $4.5 \mathrm{~min}$ at $72^{\circ} \mathrm{C}$. The primer annealing temperature $\left(55^{\circ} \mathrm{C}\right)$ was reduced by $1{ }^{\circ} \mathrm{C}$ in each cycle.
The next 25 cycles also followed the same thermal profile except the annealing temperature was held constant at $45^{\circ} \mathrm{C}$. There was a hold of $1 \mathrm{~min}$ at $72^{\circ} \mathrm{C}$ for the final extension. In order to verify the reliability and repeatability of the RAPD, $10 \%$ of the total samples were duplicated. If each pair of duplicated samples failed to produce identical bands, the gel was discarded. There was always one blank sample as a negative control, which contained all reagents for the PCR reaction, except the DNA template.

The PCR product was separated by electrophoresis in a $1.85 \%$ agarose gel in TBE buffer ( $89 \mathrm{~mm}$ Tris, $89 \mathrm{~mm}$ Borate, and 2.5 mM EDTA), stained with ethidium bromide, and viewed under ultraviolet light. The computer software alpha imager gel documentation system (Alpha Inno-tech Corp., St. San Leandro, Calif.) generated a digital image of the gel. The presence or absence of RAPD bands was scored manually in a gel image analysis software, RFLP-Pro (DNA Pro Scan, Nashville, Tenn.). If a RAPD band was present in a particular accession, it was scored as "1"; if the band was not present it was scored as " 0 ." The frequency for each RAPD marker was calculated as a proportion of the presence of a particular RAPD marker, divided by the total number of accessions included in the analysis for that species.

Distribution of RAPD frequency among the accessions of each species was plotted on a graph. The unweighted pair-group with arithmetic averages (UPGMA) method was used in the cluster analysis (Sneath and Sokal, 1973), which was available in the computer software numerical taxonomy and multivariate analysis system, NTSYS (Rohlf, 1998). The similarity coefficient for all possible pairwise combinations was calculated using NTSYS. The Simqual program of NTSYS uses an algorithm to calculate the Dice similarity coefficient for all possible pairwise combinations (Dice, 1945). The value of similarity coefficient lies between " 0 " and "1." The pairwise similarity coefficient matrix was used for the cluster analysis using UPGMA method. The result of the cluster analysis was represented in the form of a dendrogram.

Hybridization study. For the hybridization study, five $C$. chinense and five $C$. frutescens accessions were selected based on the results of the RAPD analysis to maximize the genetic distance among the hybridizing parent. All possible combinations including reciprocal hybridizations were made. The hybridizations were accomplished in a climate-controlled greenhouse. The flowers were emasculated at the white bud stage. A fresh opened flower was detached from the male parent and the dehisced anthers were rubbed on the stigma surface of the emasculated flower. Initially five hybridizations per combination were attempted, but if the flower aborted after pollination, the hybridization was repeated. Up to 15 hybridizations were made for some combinations. The mature fruits were harvested and the number of seeds set in each fruit was recorded.

Analysis of $\mathbf{F}_{1}$ Plants. The $\mathrm{F}_{1}$ seeds obtained from the hybridizations were germinated in the laboratory. The seedlings were transplanted into pots in the greenhouse. Three $F_{1}$ plants from each hybrid combination were selected for pollen viability testing. Three flowers prior to anthesis were collected from each plant. Anthers were carefully removed with forceps and placed on a microscope slide. A drop of $0.75 \%$ aceto-carmine (Carolina Biological Supply Co., N.C.) was added to each slide. The anthers were ruptured with the forceps to release the pollen grains. The slide was briefly exposed to a flame and spread with forceps to allow better staining. The total number of pollen grains and the total number of viable pollen grains in a microscope field were counted. Three counts were taken per slide. 
Table 1. List of Capsicum frutescens and C. chinense accessions used in the RAPD analysis.

\begin{tabular}{|c|c|c|c|c|c|}
\hline Accession ID & Origin ${ }^{y}$ & Species & Accession ID & Originy & Species \\
\hline PIz 939497 & Costa Rica & C. frutescens & NMCA 40029 & Columbia & C. frutescens \\
\hline PI 439498 & Costa Rica & C. frutescens & NMCA 40028 & SA218 & C. frutescens \\
\hline PI 439502 & Costa Rica & C. frutescens & Greenleaf Tabasco & United States & C. frutescens \\
\hline PI 439506 & Cuba & C. frutescens & PI 213918 & Bolivia & C. chinense \\
\hline PI 439508 & El Salvador & C. frutescens & Grief 9269 & Costa Rica & C. chinense \\
\hline PI 439509 & Fiji & C. frutescens & Grief 9304 & Colombia & C. chinense \\
\hline PI 439514 & Mexico & C. frutescens & PI 215734 & Peru & C. chinense \\
\hline PI 260479 & Peru & C. frutescens & PI 224424 & Costa Rica & C. chinense \\
\hline PI 273427 & United States & C. frutescens & Grief 9238 & Costa Rica & C. chinense \\
\hline PI 281347 & India & C. frutescens & Grief 9302 & Colombia & C. chinense \\
\hline PI 281396 & Mexico & C. frutescens & PI 224446 & Costa Rica & C. chinense \\
\hline PI 355395 & Ecuador & C. frutescens & PI 241668 & Ecuador & C. chinense \\
\hline PI 355808 & Ecuador & C. frutescens & PI 238052 & Peru & C. chinense \\
\hline PI 358811 & Malaysia & C. frutescens & PI 238047 & Guyana & C. chinense \\
\hline PI 358968 & Ecuador & C. frutescens & PI 593612 & United States & C. chinense \\
\hline PI 238059 & Guatemala & C. frutescens & PI 439455 & Peru & C. chinense \\
\hline PI 238060 & Suriname & C. frutescens & PI 439448 & Peru & C. chinense \\
\hline PI 439492 & Brazil & C. frutescens & PI 439447 & Peru & C. chinense \\
\hline PI 439490 & Brazil & C. frutescens & PI 439442 & Peru & C. chinense \\
\hline PI 439489 & Brazil & C. frutescens & PI 439426 & Costa Rica & C. chinense \\
\hline PI 39488 & Bolivia & C. frutescens & PI 439476 & United States & C. chinense \\
\hline PI 439494 & Costa Rica & C. frutescens & PI 439463 & Peru & C. chinense \\
\hline PI 439493 & Colombia & C. frutescens & PI 439462 & Peru & C. chinense \\
\hline PI 439491 & Brazil & C. frutescens & PI 439460 & Peru & C. chinense \\
\hline PI 224416 & Costa Rica & C. frutescens & PI 438614 & Mexico & C. chinense \\
\hline PI 195296 & Guatemala & C. frutescens & PI 438630 & Mexico & C. chinense \\
\hline PI 593613 & United States & C. frutescens & PI 438648 & Mexico & C. chinense \\
\hline PI 586675 & United States & C. frutescens & PI 438643 & Mexico & C. chinense \\
\hline PI 441686 & Brazil & C. frutescens & PI 260477 & Peru & C. chinense \\
\hline PI 441653 & Brazil & C. frutescens & PI 260471 & Peru & C. chinense \\
\hline PI 441647 & Brazil & C. frutescens & PI 260558 & Peru & C. chinense \\
\hline PI 441645 & Brazil & C. frutescens & PI 260522 & Peru & C. chinense \\
\hline PI 441642 & Brazil & C. frutescens & PI 260492 & Bolivia & C. chinense \\
\hline Grif 9331 & Costa Rica & C. frutescens & PI 315010 & Peru & C. chinense \\
\hline PI 593614 & Philippines & C. frutescens & PI 315019 & Peru & C. chinense \\
\hline NMCA 40040 & Zimbabwe & C. frutescens & PI 315021 & Peru & C. chinense \\
\hline NMCA 40035 & Ecuador & C. frutescens & PI 315026 & Peru & C. chinense \\
\hline NMCA 40030 & Columbia & C. frutescens & $\begin{array}{l}\text { zUSDA, Plant Int } \\
\text { yCountry from wh }\end{array}$ & $\begin{array}{l}\text { Number. } \\
\text { laterial was ori }\end{array}$ & ted. \\
\hline
\end{tabular}

\section{Results}

MoRPHOLOGICAL CHARACTERIZATION. Morphological characterization was completed for a total 301 accessions. Capsicum frutescens and $C$. chinense accessions were assigned to the corresponding species, based on morphological characteristics. According to the morphological characteristics, $70 \%$ of the accessions agreed with the species assignment at the germplasm repository. Among the accessions assigned to $C$. frutescens in the germplasm repository, $8 \%$ belonged to $C$. chinense after field observations. Similarly, among the $C$. chinense accessions, $9 \%$ were found to belong to $C$. frutescens. In addition, $8 \%$ of the accessions possessed an intermediate phenotype, making it difficult to be assigned to either $C$. frutescens or $C$. chinense species based morphology.

Variations were observed on fruit related characteristics such as fruit shape, size, and color. There were some accessions in $C$. frutescens that had large, persistent (nondeciduous) fruits. Accessions PI 439498, PI 439502, PI 238059, PI 238060, and PI

195296 fell into this category. These accessions were originally collected in Costa Rica and Guatemala. The large, pendent fruit accessions suggest that some accessions of $C$. frutescens exist in a domesticated form in addition to the cultivated 'Tabasco'.

Discriminant FUnCtion ANALYSIS. Discriminant function analysis evaluated the ability of diagnostic characters to classify

Table 2. Results of discriminant analysis in Capsicum using calyx constriction and flower position.

\begin{tabular}{lccc}
\hline Species & C. chinense & C. frutescens & Total \\
\hline From & & & \\
C. chinense & $32^{\mathrm{z}}$ & 2 & 34 \\
& $(94.12)^{\mathrm{y}}$ & $(5.88)$ & $(100.00)$ \\
C. frutescens & 2 & 38 & 40 \\
& $(5.00)$ & $(95.00)$ & $(100.00)$ \\
Total & 34 & 40 & 74 \\
& $(45.95)$ & $(54.05)$ & $(100.00)$ \\
\hline
\end{tabular}

${ }^{\text {zNumber of observations. }}$

yPercent classified into species. 

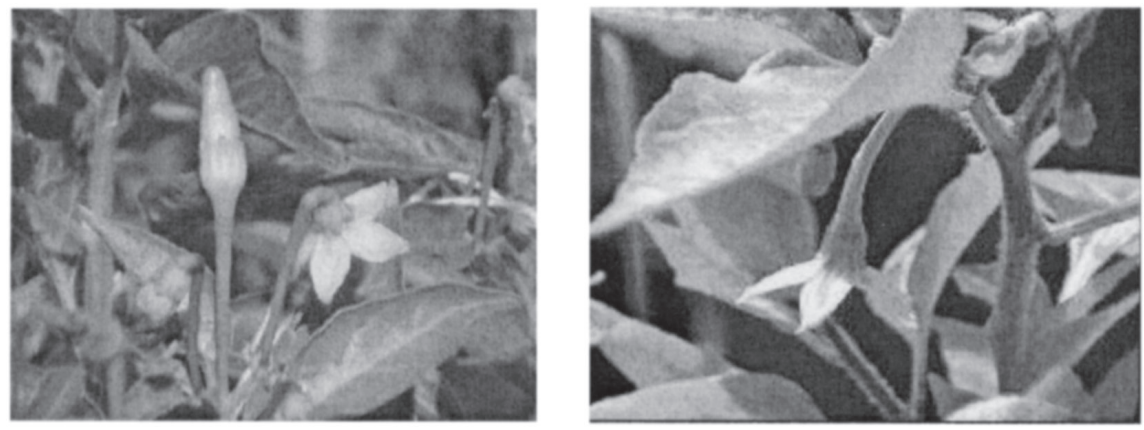

Fig. 1. Flower position as a diagnostic character to identify Capsicum frutescens and C.chinense. Capsicum frutescens (left) produces erect flowers and C. chinense (right) produces pendent flowers.

the accessions to their corresponding species. From this analysis, calyx constriction and flower position were found to be the most useful combination of diagnostic characters to differentiate the two species (Table 2). When these two characteristics were used in the analysis, $95 \%$ of the $C$. frutescens accessions could be correctly identified. Similarly, $94 \%$ of the $C$. chinense accessions could be correctly identified with the same two diagnostic characters.

The character erect flower position (Fig. 1) was present in $98 \%$ of the $C$. frutescens accessions and only in $8 \%$ of the $C$. chinense accessions. The second character separating these two species was the presence of a distinct circular constriction between the pedicel and the calyx in $C$. chinense. Calyx constriction was present in more than $92 \%$ of the $C$. chinense accessions, whereas in $C$. frutescens it was found in only $5 \%$ of the accessions.

Five percent of the $C$. chinense accessions had a single flower per axil, $5 \%$ had a mixture of single and double flowers per axil on the same plant, and $90 \%$ had two or more flowers per axil. Among the $C$. frutescens accessions, $57 \%$ of the plants had a single flower per axil, $33 \%$ had a single and two flowers, and $10 \%$ had two or more flowers per axil.

Distribution OF RAPD MARKERS. The PCR method generated RAPD markers among the accessions of $C$. frutescens and C. chinense (Fig. 2). The RAPD markers varied in size from 200 bp to 2000 bp. Using 13 primers, 106 reproducible polymorphic RAPD markers were obtained among the 75 accessions.

The majority of RAPD markers were found to be present either in a very few accessions or in the majority of the accessions in both species (bimodal distribution). If a RAPD marker frequency was greater than 0.95 in one species and $<0.05$ in the other species, that marker was considered as fixed (Wiens and
Servedio, 2000). Two RAPD markers were found to be fixed in $C$. frutescens $\left(\mathrm{OPA} 7_{750}\right.$ and OPM9 ${ }_{1950}$ ) and three RAPD markers were found to be fixed in $C$. chinense (OPAA $11_{1225}$, OPAA $11_{700}$, and $\mathrm{OPC}_{320}$ ). Furthermore, $C$. chinense had 25 unique RAPD markers that were not present in $C$. frutescens. However, 17 of these unique RAPD markers in $C$. chinense had a frequency of $<0.05$. Capsicum frutescens had seven unique RAPD markers, but five of them had a frequency of $<0.05$.

GENETIC SIMILARITY AND CLUSTER ANALYSIS. The genetic similarities within a species and between the species were calculated by averaging the Dice's similarity coefficient for pairwise comparison. The average genetic similarity between $C$. frutescens and $C$. chinense was 0.38 , whereas the average genetic similarity within $C$. frutescens was 0.85 and within $C$. chinense was 0.86 .

The cluster analysis results were graphically represented in the form of a dendrogram (Fig. 3). There were two clusters, one containing all the $C$. frutescens accessions, and the other containing all the $C$. chinense accessions. The RAPD-based cluster analysis placed 'Greenleaf Tabasco' in the $C$. frutescens group indicating high genetic similarity (Fig. 3). But, it was distinctly separated from rest of the $C$. frutescens accessions occupying an intermediate position between $C$. frutescens and $C$. chinense group.

Sexual compatibility. In the sexual compatibility study, five accessions of $C$. frutescens (PI 439497, PI 439498, PI 939489, PI 441642, and PI 195296) and five accessions of C. chinense (PI 213918, Grif 9269, Grif 9302, PI 438648, and PI 315026) were tested. A total of 819 hybridizations were performed, 352 of which were between species, and 467 of which were within species. From 93 hybridization combinations, 87 combinations developed fruits with seeds, and six combinations developed fruits without seeds. The paired $t$ test of reciprocal hybridization was not significant $(P$ $=0.80$ ), indicating the absence of unidirectional incompatibility between these two species. Selfing in $C$. frutescens gave only a $15 \%$ fruit set, whereas the hybridization among the accessions of C. frutescens produced a $36 \%$ fruit set. The difference between selfing and sibbing the plants indicates the presence of a weak self-incompatibility system in $C$. frutescens.

Comparison OF FERTILITY IN $\mathbf{F}_{1}$. The pollen viability of $F_{1}$ plants derived from the interspecific hybridizations was very low as compared to the intraspecific hybrid plants. The pollen grains

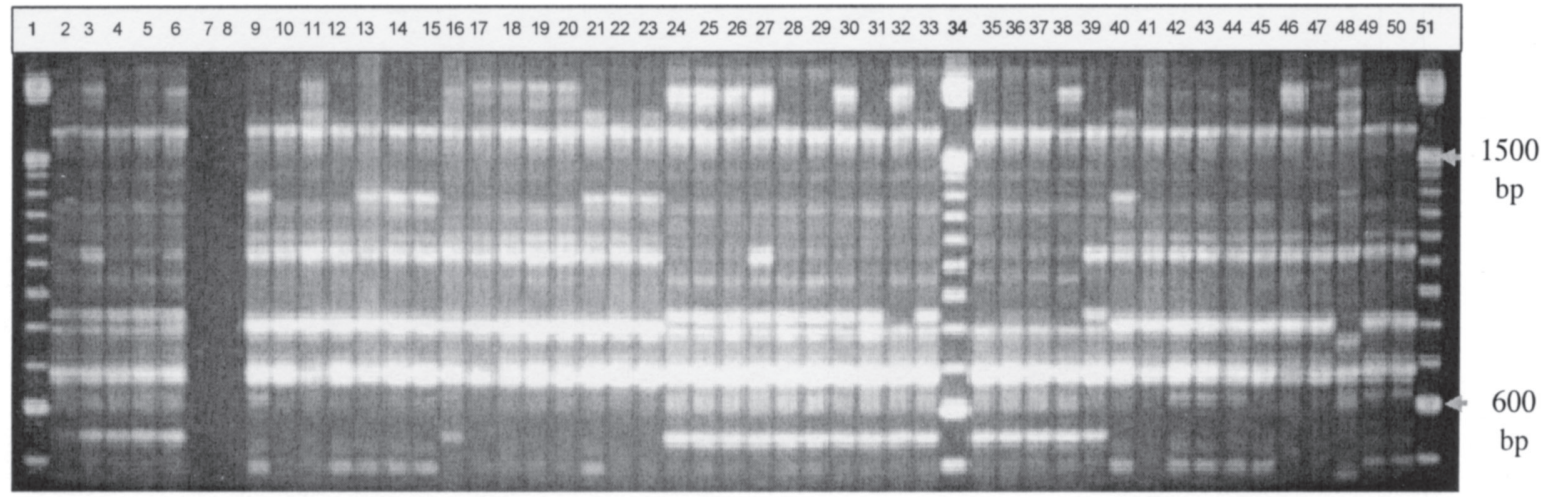

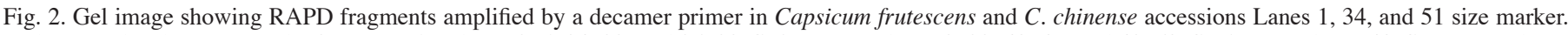

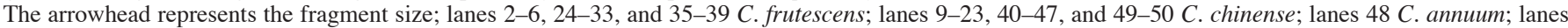
7-8 control. 


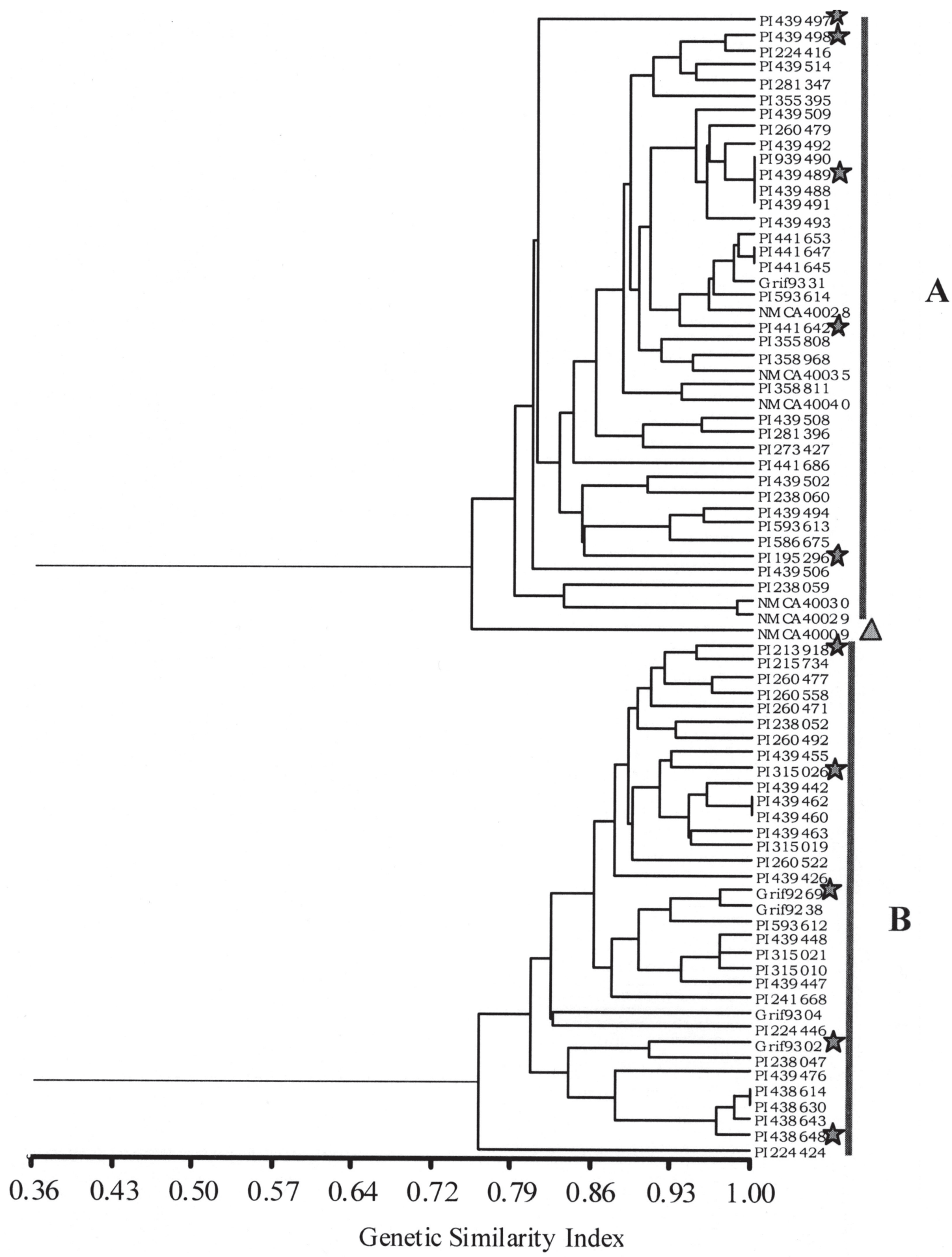

Fig. 3. Dendrogram showing genetic relationship among the accessions of Capsicum frutescens and C. chinense as revealed by RAPD markers. A = C. frutescens; $\mathbf{B}=C$. chinense $\Delta=$ Greenleaf Tabasco; $\$$ = included in hybridization .

of $C$. frutescens and $C$. chinense were similar in shape, size, and appearance under the microscope. The aceto-carmine staining technique successfully discriminated normal, viable pollen from nonviable pollen. The viable pollen appeared as large, smooth, bright red, turgid, and triangular structures (Fig. 4). Dead pollen grains appeared very small with a wrinkled surface, probably from the result of cell collapse. Pollen viability was $28 \%$ in interspecific hybrid plants, and $75 \%$ in intraspecific hybrids.

\section{Discussion}

In most cases, Capsicum frutescens and $C$. chinense could be identified by the combination of two diagnostic morphological characters: calyx constriction and flower position. The presence of species-specific RAPD markers also provided evidence that fixed differences exist between the two species at the molecular level. Capsicum frutescens and $C$. chinense represent two diag- 

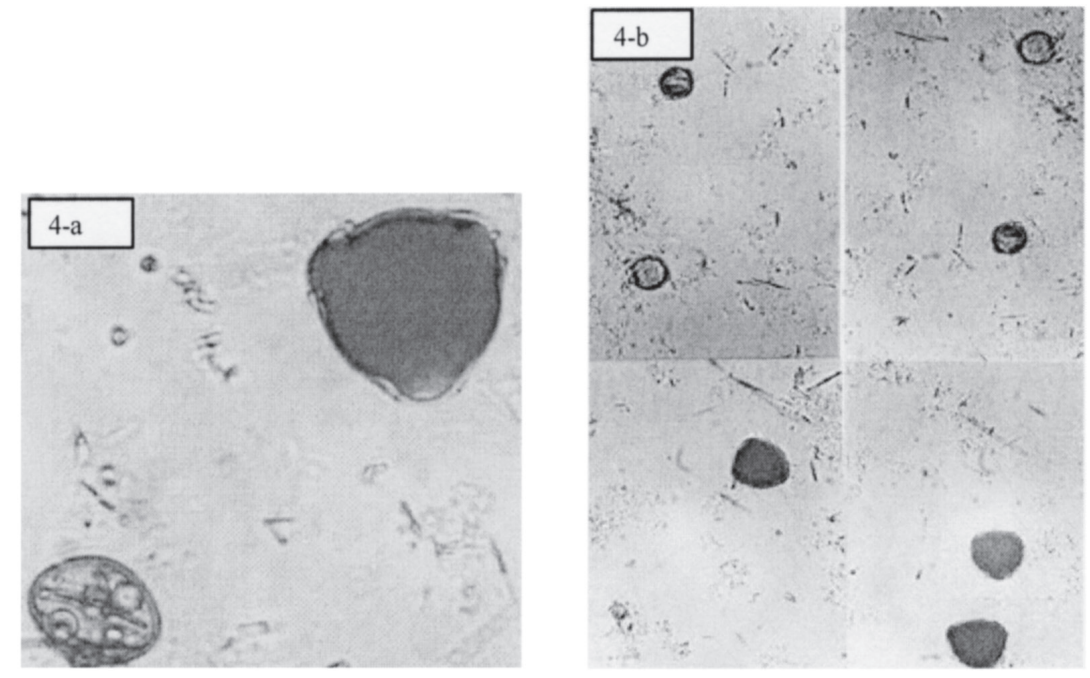

into another through the process of interspecific hybridization followed by successive backcrosses to one of the parents (Anderson, 1949). Natural hybridizations between two species have been reported in many plant species such as in Helianthus L. (Rieseberg, 2000), Iris L. (Arnold and Bennett, 1993), Pinus L. (Wang et al., 1990), and Stephanomeria Nutt. (Gallez and Gottlieb, 1982). The cultivar Greenleaf Tabasco offered an excellent example of introgression. This particular cultivar was developed by interspecific hybridization between $C$. frutescens and $C$. chinense followed by repeated backcrossing to $C$. frutescens (Greenleaf et al., 1970). 'Greenleaf Tabasco' resembles $C$. frutescens, but also possesses a few morphological characters of $C$. chinense. The RAPD analysis revealed that 'Greenleaf Tabasco' indeed possessed some DNA from $C$. chinense, as indicated by the presence of $C$. chinense specific RAPD markers. Thus, the Capsicum accessions with intermediate phenotypes represent introgressive hybridization, not intraspecific variation.

nosable clusters based on shared genetic markers. The result of the cluster analysis represented in the form of a dendrogram has clearly separated each species into separate clusters. The RAPD data did not detect any intergradations between the members of $C$. frutescens and $C$. chinense even though this phenomenon has been previously reported (Jensen et al., 1979; McLeod et al., 1983; Pickersgill, 1988). An explanation for such intergradation could be the selection of plant materials. If interspecific hybrids had been included in this study, then intergradation may have been seen. In addition, some $C$. chinense accessions in the past have been mislabeled as $C$. frutescens because the species name, $C$. chinense, was not being used.

The genetic similarity within $C$. frutescens and $C$. chinense accessions was greater than the genetic similarity between the $C$. frutescens and $C$. chinense accessions. The average genetic similarity coefficient for the pairwise comparisons between the accessions of $C$. frutescens and $C$. chinense was 0.38 . Similar results have been reported in other plant species. In Amaranthus, the average interspecific genetic similarity was 0.36 as estimated by RAPD markers (Chan and Sun, 1997). The average genetic similarity between the wild and cultivated species of Trifolium L. was 0.35, when calculated from isozyme markers (Lange and SchifinoWittmann, 2000). When intraspecific genetic variation was compared, Capsicum frutescens and $C$. chinense possessed similar levels of genetic variation. The average genetic similarity coefficient among the accessions of $C$. frutescens was 0.85 and 0.86 among $C$. chinense. Accessions of $C$. annuum var. annuum from the center of diversity, i.e., Mexico, have a lower average genetic similarity of 0.75 (Baral and Bosland, 2002).

The RAPD analysis did not support the hypothesis that $C$. frutescens is the primitive form and ancestor of $C$. chinense (Eshbaugh et al., 1983). If $C$. chinense was derived from $C$. frutescens, $C$. chinense accessions would form a cluster nested within $C$. frutescens. However, the data clearly showed parallel genetic diversity in $C$. chinense accessions, which was independent of $C$. frutescens.

Capsicum chinense and $C$. frutescens accessions with intermediate phenotypes can be explained by introgressive hybridization. With introgressive hybridization, genes of one species move
Capsicum frutescens and $C$. chinense are reproductively isolated. The development of partial sterility in the interspecific hybrid progenies of $C$. frutescens and $C$. chinense provided evidence for reproductive isolation. The pollen viability of $F_{1}$ progenies derived from the interspecific hybridizations was $28 \%$, whereas the pollen viability of the $\mathrm{F}_{1}$ progenies derived from the intraspecific hybridization was $75 \%$. In addition, the interspecific hybrid plants generally had lower fruit set. Embryo abortions were clearly visible as small brown scars on the placental surface of the fruits. Some plants even failed to produce fruit with seeds. The reduced pollen viability of interspecific hybrids has been reported in other crops. The pollen viability of the interspecific hybrid between Nuphar microphylla Fernald and N. variegata Engelm. ex Clinton was 23\% (Padgett et al., 1998). The pollen viability of interspecific hybrid among Helianthus species ranged from $11 \%$ to $42 \%$. Thus, there appears to be a genetically controlled mechanism(s) that is preventing gene flow between $C$. frutescens and $C$. chinense.

Morphological observations, RAPD-based cluster analysis, and sexual compatibility provided evidence that $C$. frutescens and $C$. chinense are distinct species. Whether the species concept used is typological, phylogenetic, or biological, they all unequivocally support the preposition that these two groups of plants are separate species. Furthermore, the RAPDs have explanatory power and can be used as a basis for establishing species identification. Two particularly important features of RAPDs were ability to do the analysis before the plant flowers, and the ability to detect interspecific introgression. The method clearly demarcates those accessions having risen from interspecific introgression, and may be useful in conservation genetics and crop evolution.

\section{Literature Cited}

Anderson, E. 1949. Introgressive hybridization. Wiley, New York. Arnold, M.L. and B.D. Bennett. 1993. Natural hybridization in Louisiana irises: Genetic variation and ecological determinants, p. 115-139. In: R.J. Harrison (ed.). Hybrid zones and evolutionary process. Oxford Univ. Press, Oxford, England.

Baral, J. and P.W. Bosland. 2002. Genetic diversity of a Capsicum germplasm collection from Nepal as determined by randomly amplified polymorphic DNA markers. J. Amer. Soc. Hort. Sci. 127:316-324. 
Chan, K.F. and M. Sun. 1997. Genetic diversity and relationships detected by isozyme and RAPD analysis of crop and wild species of Amaranthus Theor. Appl. Genet. 95:865-873.

Dice, L.R. 1945. Measures of the amount of ecological association between species. Ecology 26:297-302.

Egawa, Y. and M. Tanaka. 1984. Cytogenetic relationships among three species of chili peppers, Capsicum chinense, Capsicum frutescens and Capsicum baccatum. Jpn. J. Breeding 34:50-56.

Eshbaugh, W.H. 1993. Peppers: History and exploitation of a serendipitous new crop discovery, p. 132-139. In: J. Janick and J.E. Simon (eds.). New crops. Wiley, New York.

Eshbaugh, W.H., S.I. Guttman, and M.J. McLeod. 1983. The origin and evolution of domesticated Capsicum species. J. Ethnobiol. 3:49-54.

Gallez, G.P. and L.D. Gottlieb. 1982. Genetic evidence for the hybrid origin of the diploid plant Stephonomeria diegensis. Evolution 36:1158-1167.

Gallego, F.J. and I. Martinez. 1997. Method to improve reliability of random amplified polymorphic DNA markers. Biotechniques 22:663-664.

Greenleaf, W.H., J.A. Martin, J.G. Lease, E.T. Sims, and L.O. Van Blaricom. 1970. Greenleaf Tabasco, A new tobacco etch virus resistant 'Tabasco' pepper variety (Capsicum frutescens L.). Alabama Agr. Expt. Sta., Auburn Univ. Ala.

Jacquin, N.J. 1776. Hortus Botanicus Vindobonensis 3 pl. 82.

Jensen, R.J., M.J. McLeod, W.H. Eshbaugh, and S.I. Guttman. 1979. Numerical taxonomic analyses of allozymic variation in Capsicum (Solanaceae). Taxon 28:315-327.

Lange, O. and M.T. SchifinoWittmann. 2000. Isozyme variation in wild and cultivated species of the genus Trifolium L. (Leguminosae). Ann. Bot. 86:339-345.

Linnaeus, C. 1753. Species plantarum. Laurentii, Salvi, Stolkholm.

Mayr, E. 1970. Populations, species and evolution: an abridgement of animal species and evolution. Harvard Univ. Press, Cambridge, Mass.

McLeod, M.J., S.I. Guttman, W.H. Eshbaugh, and R.E. Rayle. 1983. An electrophoretic study of the evolution in Capsicum (Solanaceae). Evolution 37:562-574.

Padgett, D.J., D.H. Les, and G.E. Crow. 1998. Evidence for the hy- brid origin of Nuphar xrubrodisca (Nymphaeaceae). Amer. J. Bot. 85:1468-1476.

Pickersgill, B. 1971. Relationships between weedy and cultivated forms in some species of chili peppers (Genus Capsicum). Evolution 25:683-691.

Pickersgill, B. 1988. The genus Capsicum: A multidisciplinary approach to the taxonomy of cultivated and wild plants. Biologisches Zentralblatt 107:381-389.

Pickersgill, B., C.B. Heiser, and J. McNeill. 1979. Numerial taxonomic studies on variation and domestication in some species of Capsicum, $\mathrm{p}$. 679-700. In: Hawkes, J.G., R.N. Lester, and A.D. Skelding (eds.). The biology and taxonomy of the Solanaceae. Academic, London.

Rieseberg, L.H. 2000. Crossing relationships among ancient and experimental sunflower hybrid lineages. Evolution 54:859-865.

Rohlf, F.J. 1998. Numerical taxonomy and multivariate analysis system (NTSYSpc): Ver. 2.0. Exeter Publ., Setauket, N.Y.

SAS Inst. 2001. SAS Software release 8.2. SAS Inst., Cary, N.C.

Shaw, F.J.F. and A.R. Khan. 1928. Studies in Indian chillies. Memoirs Dept. Agr. India, Bot. Ser. 16:59-82.

Smith, P.G. and C.B. Heiser, Jr. 1957. Breeding behavior of cultivated peppers. Proc. Amer. Soc. Hort. Sci. 70:286-290.

Sneath, P.H.A. and R.R. Sokal. 1973. Numerical taxonomy. W.H. Freeman, San Francisco.

Standley, P.C. 1931. Flora of the Lancetilla Valley, Honduras. Field Museum of Natural History, Bot. Ser. 10.

Wang, X.R., A.E. Szmidt, A. Lewandowski, and G.R. Wang. 1990. Evolutionary analysis of Pinus densata (Masters): A putative tertiary hybrid. Theor. Appl. Gen. 80:635-640.

Wiens, J.J. and M.R. Servedio. 2000. Species delimitation in systematics: inferring diagnostic differences between species. Proc. Royal Soc. London, Ser. B 267:631-636.

Williams, J.G.K., M.K. Hanafey, J.A. Rafalski, and S.V. Tingey. 1993. Genetic analysis using random amplified polymorphic DNA markers. Methods Enzymol. 218:704-740.

Votava, E.J. and P.W. Bosland. 2002. A cultivar by any other name: Genetic variability in an heirloom bell pepper-Capsicum annuum $\mathrm{L}$. var. annuиm 'California Wonder'. HortScience 37:1100-1102. 